\title{
Bronchogenic carcinoma: incidence of metastases to normal sized lymph nodes
}

\author{
Takeshi Arita, Tatsuya Kuramitsu, Mitsutoshi Kawamura, Tsuneo Matsumoto, \\ Naofumi Matsunaga, Kazuro Sugi, Kensuke Esato
}

\begin{abstract}
Background - The incidence of metastases to mediastinal lymph nodes was evaluated in patients with normal sized mediastinal nodes on the computed tomographic (CT) scan who underwent thoracotomy. The use of hilar lymph nodes in predicting mediastinal lymph node metastases was also assessed.

Methods - Ninety patients with non-small cell lung cancer who later underwent thoracotomy were prospectively examined by CT scanning. Lymph nodes with a short axis diameter of $10 \mathrm{~mm}$ or more were considered abnormal.

Results - Mediastinal lymph node metastases were present at thoracotomy in 19 patients $(21 \%)$. In 14 these lymph node metastases were misdiagnosed because the nodes were normal in size on the $C T$ scan. In only one of the 19 patients with $\mathrm{N} 2$ nodes was an N1 lymph node enlarged, and four of the 19 patients with $\mathrm{N} 2$ nodes had metastases to these mediastinal nodes without N1 disease ("skipping metastases").

Conclusions - Metastases in normal sized nodes seen on the CT scan are a major problem in staging. Hilar lymph nodes did not help to predict reliably the presence or absence of metastases to the mediastinal lymph nodes.
\end{abstract}

(Thorax 1995;50:1267-1269)

Keywords: computed tomographic (CT) scanning, lung neoplasm, lymph node.

Accurate staging of lung cancer, including the extent of local and distant disease, is essential to determine the resectability and prognosis. ${ }^{12}$ Preoperative assessment of mediastinal lymph nodes both clinically and radiologically is important, but the value of computed tomographic (CT) staging of bronchogenic carcinoma remains controversial. ${ }^{3}$ Although most early studies reported that CT scanning had a high sensitivity for detecting mediastinal lymph node metastases, ${ }^{4-6}$ more recent studies suggest that CT scanning has a low sensitivity..$^{7-9}$ In a study by McKenna et al, ${ }^{7}$ in which total nodal sampling was performed, a lower sensitivity $(60 \%)$ was reported for the detection of mediastinal lymph node metastases with CT scanning than in earlier studies.

In general, the smaller the node size chosen to separate malignant tissue from benign, the higher the sensitivity and the lower the specificity. The frequency of metastases to normal sized mediastinal lymph nodes is a subject of debate that directly affects the sensitivity of CT scanning, although it has been claimed that metastasis to normal sized lymph nodes (incidence $7 \%$ ) was not a major problem in CT staging of non-small cell lung cancer. ${ }^{10}$

We have further evaluated the incidence of metastases to normal sized nodes in patients with non-small cell lung cancer who underwent thoracotomy. Furthermore, we have assessed the use of hilar lymph nodes in predicting mediastinal lymph node metastases.

\section{Methods}

Between April 1989 and October 1994243 patients with primary lung cancer were assessed in our department. Of these we prospectively studied by CT scanning 90 patients ( 66 men) who later underwent thoracotomy for primary lung cancer. Their mean age was 64 years (range 40-79). Selection criteria for thoracotomy in our institution were as follows: (1) no visible direct mediastinal invasion by the tumour on the CT scan; (2) no enlarged contralateral mediastinal nodes on the CT scan; and (3) no known distant metastases.

Patients with stage III B and stage IV disease at preoperative staging did not undergo thoracotomy. CT scans were performed at least three weeks before thoracotomy with a TCT-900S machine (Toshiba, Tokyo, Japan). Contiguous $1 \mathrm{~cm}$ thick sections were obtained at $1 \mathrm{~cm}$ intervals from the lung apices to at least the adrenal glands. CT scanning was initiated about 30 seconds after start of intravenous power injection of $100 \mathrm{ml}$ contrast material (Iopamidol 300, Bracco Industria Chemica, Milan, Italy) at the rate of $2 \mathrm{ml} / \mathrm{s}$. Standard scanning parameters $(120 \mathrm{kVp}, 120-250 \mathrm{~mA}$, $512 \times 512$ matrix, 1 second scanning time) were used, depending on the size of the patient. CT scans were reviewed at levels appropriate for the mediastinum (level, $50 \mathrm{HU}$; window, $300 \mathrm{HU}$ ). The CT scans were independently interpreted by three radiologists (TA, TK, TM). When the analysis was not unanimous a consensus was reached after discussion. Mediastinal lymph nodes were localised according to the lymph node mapping scheme of the Japanese Lung Cancer Society ${ }^{11} 12$ derived from the proposition by Naruke et $a l^{13}$ which is used internationally. Lymph nodes were measured along the short axis on the transverse images and were considered abnormal if they were $10 \mathrm{~mm}$ or more in diameter. ${ }^{14}$ Furthermore, lymph nodes which none of the three radiologists could detect on the CT images were defined as undetectable 
nodes if found to be metastatic at thoracotomy.

All patients underwent thoracotomy with dissection of the mediastinal lymph nodes. Mediastinoscopy was not performed before thoracotomy in our institution. However, mediastinoscopy was performed to evaluate lymph nodes on the contralateral side of the mediastinum at thoractomy regardless of staging. If the lymph nodes were accessible, a biopsy specimen was taken. Furthermore, R2b station resection was performed regardless of staging (stage I, II, IIIA). ${ }^{11}$ At right thoracotomy all accessible lymph nodes in the superior mediastium - that is, the superior mediastinal, paratracheal, anterior mediastinal, pretracheal and retrotracheal, and tracheobronchial nodes - and inferior mediastinum that is, the subcarinal, paraoesophageal, and pulmonary ligament nodes - were resected together with the surrounding fat tissue. At left thoractomy all accessible lymph nodes in the superior mediastinum - that is, the superior mediastinal, anterior mediastinal, pretracheal and retrotracheal, tracheobronchial, subaortic, and para-aortic nodes - and inferior mediastinum - that is, the subcarinal, paraoesophageal, and pulmonary ligament nodes were resected. At thoracotomy, hilar (station 10), interlobar (station 11 on the left side, station $11 \mathrm{~s}$ on the right upper lobectomy, $11 \mathrm{i}$ at right lower lobectomy, $11 \mathrm{~s}$ plus $11 \mathrm{i}$ at middle lobectomy) and lobar nodes (station 12) were resected. Specimens from the maximal cut surface of each resected lymph node were routinely stained with haematoxylin and eosin.

\section{Results}

Of the 90 bronchogenic carcinomas 61 were adenocarcinomas, 25 squamous cell, three large cell, and one adenosquamous. Mediastinal lymph node metastases were present in 19 patients $(21 \%)-14$ with adenocarcinoma and five with squamous cell carcinoma. Four hundred and seventy five mediastinal nodal stations (443 at thoracotomy, 32 at mediastinoscopy) and 162 hilar nodal stations (156 at thoracotomy, six at mediastinoscopy) were sampled (mean of $7 \cdot 1$ stations per patient) (table 1). In 40 of the 475 mediastinal nodal stations $(8.5 \%)$ and in 31 of the 162 hilar nodal stations $(19 \%)$ metastatic lymph nodes were demonstrated histopathologically (table 1 ).

A total of 1443 mediastinal lymph nodes and 542 hilar lymph nodes were resected at thoracotomy (mean $22 \cdot 1$ nodes per patient, $3 \cdot 3$ nodes per nodal station). Sixty eight of the resected mediastinal lymph nodes $(4 \cdot 7 \%)$ and 63 of the resected hilar lymph nodes $(11 \cdot 6 \%)$ contained malignant tissue.

Enlarged N2 lymph nodes were seen on the CT scan in five of 19 patients with mediastinal lymph node metastases (26\%). In the remaining 14 patients normal sized N2 nodes or undetectable N2 nodes were seen (table 2). Furthermore, in six of the 19 patients metastatic tumour was present, not in the enlarged nodes, but in nodes that were not identified on the CT scan in different mediastinal nodal stations. In addition, four of the 19 patients
Table 1 Number of fapanese Lung Cancer Society nodal stations sampled in 90 patients

\begin{tabular}{lcc}
\hline $\begin{array}{l}\text { Node } \\
\text { station }\end{array}$ & $\begin{array}{l}\text { No. of sampled } \\
\text { nodal stations }\end{array}$ & $\begin{array}{l}\text { No. of stations with } \\
\text { metastases }\end{array}$ \\
\hline 1 & 35 & 0 \\
$2 \mathrm{R}$ & 33 & 1 \\
$2 \mathrm{~L}$ & 23 & 0 \\
3 & 79 & 9 \\
$4 \mathrm{R}$ & 46 & 6 \\
$4 \mathrm{~L}$ & 35 & 4 \\
5 & 33 & 5 \\
6 & 40 & 1 \\
7 & 69 & 7 \\
8 & 41 & 3 \\
9 & 41 & 4 \\
Subtotal & 475 & 40 \\
& & 4 \\
$10 \mathrm{R}$ & 30 & 4 \\
$10 \mathrm{~L}$ & 30 & 3 \\
11R & 27 & 6 \\
11L & 27 & 6 \\
12R & 25 & 8 \\
12L & 23 & 71 \\
Subtotal & 162 & \\
Total & 637 & \\
\hline
\end{tabular}

Table 2 Analysis of 19 patients and 40 stations with mediastinal lymph node metastases using the size of lymph nodes on the CT scan

\begin{tabular}{lcc}
\hline $\begin{array}{l}\text { Status of N2 nodes } \\
\text { by size }\end{array}$ & $\begin{array}{l}\text { No. of nodal } \\
\text { stations }\end{array}$ & $\begin{array}{l}\text { No. of } \\
\text { cases }\end{array}$ \\
\hline Normal sized N2 nodes & $11(27 \cdot 5 \%)$ & $7(37 \%)$ \\
Undetectable N2 nodes & $23(57 \cdot 5 \%)$ & $7(37 \%)$ \\
Enlarged N2 nodes & $6(15 \%)$ & $5(26 \%)$ \\
Total & $40(100 \%)$ & $19(100 \%)$ \\
\hline
\end{tabular}

Table 3 Computed tomographic status of hilar lymph nodes of 19 patients with mediastinal lymph node metastases

\begin{tabular}{|c|c|}
\hline $\begin{array}{l}\text { Status of hilar } \\
\text { nodes on } C T \text { scan }\end{array}$ & $\begin{array}{l}\text { No. of } \\
\text { cases }\end{array}$ \\
\hline $\begin{array}{l}\text { Normal sized or undetectable N1 nodes } \\
\text { Enlarged N1 nodes } \\
\text { Without N1 nodes }\end{array}$ & $\begin{array}{rr}14 & (74 \%) \\
1 & (5 \%) \\
4 & (21 \%)\end{array}$ \\
\hline Total & $19(100 \%)$ \\
\hline
\end{tabular}

with $\mathrm{N} 2$ nodes had metastases to mediastinal lymph nodes without metastases to hilar lymph nodes ("skipping metastases"). In only one of these patients was an enlarged N1 node seen on the CT scan (table 3). In three of the 71 patients without N2 nodes the hilar lymph nodes were enlarged.

Biopsy samples were taken at mediastinoscopy from 38 contralateral stations. Three metastatic stations were found. However, enlarged lymph nodes were not seen in those stations on the CT scan.

The overall sensitivity, specificity, and accuracy of CT scanning in detecting mediastinal lymph node metastases were $58 \%, 87 \%$, and $81 \%$, respectively.

The presence of mediastinal invasion was seen in four of the 90 patients at thoracotomy. Mediastinal invasion was not evaluated by mediastinoscopy.

\section{Discussion}

We have studied 90 patients with non-small cell lung cancer. Nodes identified as being $10 \mathrm{~mm}$ or more in diameter on the CT scan were considered suggestive of malignant involvement. These findings were compared with 
results of operative dissection. In 19 of the patients mediastinal lymph node metastases were found, in only one of whom an N1 node was enlarged. Furthermore, in 14 of 19 patients with N2 nodes mediastinal lymph node metastases were misdiagnosed, and four patients had N2 nodes without N1 nodes ("skipping metastases"). The status of hilar lymph nodes was not therefore predictive of $\mathrm{N} 2$ involvement, and metastases in normal sized nodes seen on the CT scan were a major problem in staging.

Kerr et $a l^{15}$ reported that malignant mediastinal lymph nodes were not larger than benign nodes and small mediastinal lymph nodes were not infrequently malignant. Their findings support our study in the frequency of metastases to small lymph nodes. Furthermore, they suggested that, in the light of their findings of considerable numbers of "small" lymph nodes containing malignant tissue, small lymph nodes potentially "missed" by the surgeon would clearly be of interest. The higher incidence of patients with metastases to normal sized nodes in our study may be due to the fact that the number of resected nodes was more extensive than that in other studies. ${ }^{8-10}$

The most important findings in our study were that in only one of the patients with $\mathrm{N} 2$ disease was an enlarged N1 node seen, and some patients had $\mathrm{N} 2$ nodes without $\mathrm{N} 1$ nodes ("skipping metastases"). These findings indicate that we are not able to predict the presence or absence of metastases to mediastinal lymph nodes by the size of the hilar lymph nodes, and the absence of metastases to hilar lymph nodes is not always connected with the absence of metastases to mediastinal lymph nodes.

Some limitations of this study must be stressed. Our data are derived from a small and selected group of patients. Furthermore, mediastinoscopy was not performed before thoracotomy so we were not able to evaluate the role of mediastinoscopy in preoperative staging. However, in three of 19 patients with $\mathrm{N} 2$ nodes, N3 nodes were seen by mediastinoscopy which were misdiagnosed because of metastases to normal sized nodes on CT scanning. Mediastinoscopy therefore seems to be more useful than CT scanning in the assessment of N3 nodes.

1 Pearson FG, DeLaure NC, Ileves R, Todd TRJ, Cooper D. Significance of positive superior mediastinal nodes dentified at mediastinoscopy in patients with resectable cancer of the lung. 7 Thorac Cardiovasc Surg 1982;83: $1-11$.

2 Ratto GB, Mereu C, Motta G. The prognostic significance of preoperative assessment of mediastinal lymph nodes in patients with lung cancer. Chest 1988;93:807-13.

3 Gallardo JFM, Naranjo FB, Cansino MT, RodoriguezPanadero F. Validity of enlarged mediastinal nodes as markers of involvement by non-small cell lung cancer. $A m$ Rev Respir Dis 1992;146:1210-2.

4 Failing LJ, Pugatch RD, Jung-Legg Y. Computed tomography scanning the mediastinum in the staging of bronchogenic carcinoma. Am Rev Respir Dis 1981;124 690-5.

5 Osborn DR, Korobkin M, Ravin CE, Putman CE, Wolfe WG, Sealy WC, et al. Comparison of plain radiography, conventional tomography, and computed tomography in detecting lymph node metastasis from lung cancer. Radiology 1982;142:157-61.

6 Baron RL, Levitt RG, Sagelss SS, White MJ, Roger CL Marberger JP. Computed tomography in preoperative evaluation of bronchogenic carcinoma. Radiology 1982; 145:727-32.

7 McKenna RJ Jr, Libshitz HZ, Mountain CE, McMurtney MJ. Roentgenographic evaluation of mediastinal nodes for preoperative assessment in lung cancer. Chest 1985; 88:206-10.

8 Staples CA, Muller NL, Miller RR, Evance KG, Nelms B. Mediastinal nodes in bronchogenic carcinoma: comparison between CT and mediastinoscopy. Radiology 1988; 167:367-72.

9 Webb WR, Gatson SC, Zerhouni E, Heelen RT, Glazer GM, Francis IR, et al. CT and MR imaging in staging non-small cell bronchogenic carcinoma: report of the Radiographic Diagnostic Oncology Group. Radiology 1991:178:705-13.

10 Gross BH, Glazer GM, Orringer MB, Spizarny DL, Flin A. Bronchogenic carcinoma metastatic to normal-sized nodes: frequency and significance. Radiology 1988;166: $71-4$.

11 Japanese Lung Cancer Society. General rule for clinical and pathological reconding of lung cancer. 3rd ed. Tokyo, Japan: Kanehara, 1987:79-85 [Japanese].

12 Watanabe Y, Shimizu J, Tsubota M, Iwa T. Mediastinal spread of metastatic lymph nodes in bronchogenic carcinoma: mediastinal nodal metastases in lung cancer Chest cinoma: mediastinal

13 Naruke T, Suemasu K, Ishikawa S. Lymph node mapping and curability at various levels of metastasis in resected lung cancer. $\mathcal{F}$ Thorac Cardiovasc Surg 1978;76:832-9.

14 Yokoi K, Okuyama A, Mori K, Tominaga K, Miyazawa N, Takizawa I, et al. Mediastinal lymph node metastasis from lung cancer: evaluation with T1-201 SPECT - comparison with CT. Radiology 1994;192:813-7.

15 Kerr KM, Lamb D, Wathen CG, Walker WS, Douglas NJ. Pathological assessment of mediastinal lymph nodes in lung cancer: implications for non-invasive mediastinal staging. Thorax 1992;47:337-41. 